\title{
Daneš theorem in complete random normed modules
}

Yujie Yang*

\section{"Correspondence:} yangyujie007@163.com

Department of Basic Course, Beijing Union University, Beijing, 100101,

P.R. China

\section{Springer}

\begin{abstract}
Based on the recent work of random metric theory, namely the Ekeland variational principle on a complete random metric space, this paper studies the Daneš theorem in a complete random normed module. In this paper, we first present the notion of finer ordering on a random normed module. Then we establish the Daneš theorem in a complete random normed module under the locally $L^{0}$-convex topology. When the base space of the random normed module is trivial, our result automatically degenerates to the classical case.
\end{abstract}

MSC: $58 \mathrm{E} 30 ; 47 \mathrm{H} 10 ; 46 \mathrm{H} 25 ; 46 \mathrm{~A} 20$

Keywords: random metric space; random normed module; locally $L^{0}$-convex topology; Ekeland variational principle

\section{Introduction}

In 1972, Daneš [1] presented the Daneš theorem. With the classical Ekeland variational principle, Brøndsted [2] gave it a new proof in 1974.

Recently, Prof. Guo Tiexin and I [3] established the Ekeland variational principle for an $\bar{L}^{0}$-valued function on a complete random metric space, where $\bar{L}^{0}$ is the set of equivalence classes of extended real-valued random variables on a probability space $(\Omega, \mathcal{F}, P)$. Based on this result, this paper establishes the Daneš theorem in a complete random normed module under the locally $L^{0}$-convex topology.

A random normed module is a random generalization of an ordinary normed space. Different from ordinary normed spaces, random normed modules possess the rich stratification structure, which is introduced in this paper. It is this kind of rich stratification structure that makes the theory of random normed modules deeply developed and also renders it the most useful part of random metric theory [4-14]. When the probability $(\Omega, \mathcal{F}, P)$ is trivial, namely $\mathcal{F}=\{\emptyset, \Omega\}$, our result reduces to the classical Daneš theorem. So our result is a nontrivial random extension.

The remainder of this article is organized as follows: in Section 2 we give some necessary definitions and in Section 3 we give the main results and proofs.

\section{Preliminary}

Throughout this paper, $(\Omega, \mathcal{F}, P)$ denotes a probability space, $K$ the real number field $R$ or the complex number field $C, N$ the set of positive integers, $\bar{L}^{0}(\mathcal{F})$ the set of equivalence classes of extended real-valued random variables on $\Omega$ and $L^{0}(\mathcal{F}, K)$ the algebra of equivalence classes of $K$-valued $\mathcal{F}$-measurable random variables on $\Omega$ under the ordinary scalar

C2014 Yang; licensee Springer. This is an Open Access article distributed under the terms of the Creative Commons Attribution License (http://creativecommons.org/licenses/by/2.0), which permits unrestricted use, distribution, and reproduction in any medium, provided the original work is properly cited. 
multiplication, addition and multiplication operations on equivalence classes, denoted by $L^{0}(\mathcal{F})$ when $K=R$.

Specially, $L_{+}^{0}(\mathcal{F})=\left\{\xi \in L^{0}(\mathcal{F}) \mid \xi \geq 0\right\}, L_{++}^{0}(\mathcal{F})=\left\{\xi \in L^{0}(\mathcal{F}) \mid \xi>0\right.$ on $\left.\Omega\right\}$.

As usual, $\xi>\eta$ means $\xi \geq \eta$ and $\xi \neq \eta$, whereas $\xi>\eta$ on $A$ means $\xi^{0}(\omega)>\eta^{0}(\omega)$ a.s. on $A$ for any $A \in \mathcal{F}$ and $\xi$ and $\eta$ in $\bar{L}^{0}(\mathcal{F})$, where $\xi^{0}$ and $\eta^{0}$ are arbitrarily chosen representatives of $\xi$ and $\eta$, respectively.

For any $A \in \mathcal{F}, A^{c}$ denotes the complement of $A, \tilde{A}=\{B \in \mathcal{F} \mid P(A \Delta B)=0\}$ denotes the equivalence class of $A$, where $\Delta$ is the symmetric difference operation, $I_{A}$ the characteristic function of $A$, and $\tilde{I}_{A}$ is used to denote the equivalence class of $I_{A}$; given two $\xi$ and $\eta$ in $\bar{L}^{0}(\mathcal{F})$, and $A=\left\{\omega \in \Omega: \xi^{0} \neq \eta^{0}\right\}$, where $\xi^{0}$ and $\eta^{0}$ are arbitrarily chosen representatives of $\xi$ and $\eta$, respectively, then we always write $[\xi \neq \eta]$ for the equivalence class of $A$ and $I_{[\xi \neq \eta]}$ for $\tilde{I}_{A}$, one can also understand the implication of such notations as $I_{[\xi \leq \eta]}, I_{[\xi<\eta]}$, and $I_{[\xi=\eta]}$.

For an arbitrary chosen representative $\xi^{0}$ of $\xi \in L^{0}(\mathcal{F}, K)$, define the two $\mathcal{F}$-measurable random variables $\left(\xi^{0}\right)^{-1}$ and $\left|\xi^{0}\right|$ by $\left(\xi^{0}\right)^{-1}(\omega)=\frac{1}{\xi^{0}(\omega)}$ if $\xi^{0}(\omega) \neq 0$, and $\left(\xi^{0}\right)^{-1}(\omega)=0$ otherwise, and by $\left|\xi^{0}\right|(\omega)=\left|\xi^{0}(\omega)\right|, \forall \omega \in \Omega$. Then the equivalence class $\xi^{-1}$ of $\left(\xi^{0}\right)^{-1}$ is called the generalized inverse of $\xi$ and the equivalence class $|\xi|$ of $\left|\xi^{0}\right|$ is called the absolute value of $\xi$. It is clear that $\xi \cdot \xi^{-1}=I_{[\xi \neq 0]}$.

Definition 2.1 ([15]) An ordered pair $(E,\|\cdot\|)$ is called a random normed space (briefly, an $R N$ space) over $K$ with base $(\Omega, \mathcal{F}, P)$ if $E$ is a linear space and $\|\cdot\|$ is a mapping from $E$ to $L_{+}^{0}(\mathcal{F})$ such that the following three axioms are satisfied:

(1) $\|x\|=0$ if and only if $x=\theta$ (the null vector of $E$ );

(2) $\|\alpha x\|=|\alpha|\|x\|, \forall \alpha \in K$ and $x \in E$;

(3) $\|x+y\| \leq\|x\|+\|y\|, \forall x, y \in E$,

where the mapping $\|\cdot\|$ is called the random norm on $E$ and $\|x\|$ is called the random norm of a vector $x \in E$.

In addition, if $E$ is left module over the algebra $L^{0}(\mathcal{F}, K)$ such that the following is also satisfied:

(4) $\|\xi x\|=|\xi|\|x\|, \forall \xi \in L^{0}(\mathcal{F}, K)$ and $x \in E$,

then such an $R N$ space is called an $R N$ module over $K$ with base $(\Omega, \mathcal{F}, P)$ and such a random norm $\|\cdot\|$ is called an $L^{0}$-norm on $E$.

Definition $2.2([16])$ Let $(E,\|\cdot\|)$ be an $R N$ module over $K$ with base $(\Omega, \mathcal{F}, P)$. For any $\varepsilon \in L_{++}^{0}$, let $B(\varepsilon)=\{x \in E \mid\|x\| \leq \varepsilon\}$ and $\mathcal{U}_{\theta}=\left\{B(\varepsilon) \mid \varepsilon \in L_{++}^{0}\right\}$. A set $G \subset E$ is called $\mathcal{T}_{c}$-open if for every $x \in G$ there exists some $B(\varepsilon) \in \mathcal{U}_{\theta}$ such that $x+B(\varepsilon) \subset G$. Let $\mathcal{T}_{c}$ be the family of $\mathcal{T}_{c}$-open subsets, then $\mathcal{T}_{c}$ is a Hausdorff topology on $E$, called the locally $L^{0}$-convex topology, denoted by $\mathcal{T}_{c}$.

Let $(E,\|\cdot\|)$ be an $R N$ module over $K$ with base $(\Omega, \mathcal{F}, P), p_{A}=\tilde{I}_{A} \cdot p$ is called the $A$-stratification of $p$ for each given $A \in \mathcal{F}$ and $p$ in $E$. The so-called stratification structure of $E$ means that $E$ includes every stratification of an element in $E$. Clearly, $p_{A}=\theta$ when $P(A)=0$ and $p_{A}=p$ when $P(\Omega \backslash A)=0$, which are both called trivial stratifications of $p$. Further, when $(\Omega, \mathcal{F}, P)$ is a trivial probability space every element in $E$ has merely the two trivial stratifications since $\mathcal{F}=\{\Omega, \emptyset\}$; when $(\Omega, \mathcal{F}, P)$ is arbitrary, every element in $E$ can possess arbitrarily many nontrivial intermediate stratifications. It is this kind of rich stratification structure of $R N$ modules that makes the theory of $R N$ modules deeply developed and also renders it the most useful part of random metric theory. 
To introduce the main results of this paper, let us first recall the following.

Definition 2.3 ([3]) Let $X$ be a Hausdorff space and $f: X \rightarrow \bar{L}^{0}(\mathcal{F})$, then:

(1) $\operatorname{dom}(f):=\{x \in X \mid f(x)<+\infty$ on $\Omega\}$ is called the effective domain of $f$.

(2) $f$ is proper if $f(x)>-\infty$ on $\Omega$ for every $x \in X$ and $\operatorname{dom}(f) \neq \emptyset$.

(3) $f$ is bounded from below (resp., bounded from above) if there exists $\xi \in L^{0}(\mathcal{F})$ such that $f(x) \geq \xi$ (accordingly, $f(x) \leq \xi$ ) for any $x \in X$.

In all the vector-valued extensions of the Ekeland variational principle, it is of key importance to properly define the lower semicontinuity for a vector-valued function [17-19]. Recently, we have found that a kind of lower semicontinuity for $\bar{L}^{0}$-valued functions is very suitable for the study of conditional risk measures.

Definition 2.4 ([16]) Let $(E,\|\cdot\|)$ be an $R N$ module over $R$ with base $(\Omega, \mathcal{F}, P)$. A function $f: E \rightarrow \bar{L}^{0}(\mathcal{F})$ is called $\mathcal{T}_{c}$-lower semicontinuous if epi $(f)$ is closed in $\left(E, \mathcal{T}_{c}\right) \times\left(L^{0}(\mathcal{F}), \mathcal{T}_{c}\right)$.

There is a kind of countable concatenation property, which is concerned with the $L^{0}$ module $E$ itself and is very important for the theory of $R N$ module. Let us recall it.

Definition 2.5 ([10]) Let $E$ be a left module over the algebra $L^{0}(\mathcal{F}, K)$. A formal sum $\sum_{n \in N} \tilde{I}_{A_{n}} x_{n}$ is called a countable concatenation of a sequence $\left\{x_{n} \mid n \in N\right\}$ in $E$ with respect to a countable partition $\left\{A_{n} \mid n \in N\right\}$ of $\Omega$ to $\mathcal{F}$. Moreover, a countable concatenation $\sum_{n \in N} \tilde{I}_{A_{n}} x_{n}$ is well defined or $\sum_{n \in N} \tilde{I}_{A_{n}} x_{n} \in E$ if there is $x \in E$ such that $\tilde{I}_{A_{n}} x=\tilde{I}_{A_{n}} x_{n}$, $\forall n \in N$. A subset $G$ of $E$ is said to have the countable concatenation property if every countable concatenation $\sum_{n \in N} \tilde{I}_{A_{n}} x_{n}$ with $x_{n} \in G$ for each $n \in N$ still belongs to $G$, namely $\sum_{n \in N} \tilde{I}_{A_{n}} x_{n}$ is well defined and there exists $x \in G$ such that $x=\sum_{n \in N} \tilde{I}_{A_{n}} x_{n}$.

Definition 2.6 ([16]) Let $E$ be a left module over the algebra $L^{0}(\mathcal{F})$ and $f$ a function from $E$ to $\bar{L}^{0}(\mathcal{F})$, then:

(1) $f$ is $L^{0}(\mathcal{F})$-convex if $f(\xi x+(1-\xi) y) \leq \xi f(x)+(1-\xi) f(y)$ for all $x$ and $y$ in $E$ and $\xi \in L_{+}^{0}(\mathcal{F})$ such that $0 \leq \xi \leq 1$ (here we make the convention that $0 \cdot( \pm \infty)=0$ and $\infty-\infty=\infty !)$.

(2) $f$ is said to have the local property if $\tilde{I}_{A} f(x)=\tilde{I}_{A} f\left(\tilde{I}_{A} x\right)$ for all $x \in E$ and $A \in \mathcal{F}$.

It is well known from [16] that $f: E \rightarrow \bar{L}^{0}(\mathcal{F})$ is $L^{0}(\mathcal{F})$-convex iff $f$ has the local property and epi $(f)$ is $L^{0}(\mathcal{F})$-convex.

\section{Main results and proofs}

Definition 3.1 Let $(E,\|\cdot\|)$ be an $R N$ module over $R$ with base $(\Omega, \mathcal{F}, P), z \in E$ and $r \in$ $L_{++}^{0}(\mathcal{F})$. Denote the $\mathcal{T}_{c^{-}}$-closed ball by

$$
B_{z}(r):=\{x \in E:\|x-z\| \leq r\} .
$$

Definition $3.2([14])$ Let $(E,\|\cdot\|)$ be an $R N$ module over $R$ with base $(\Omega, \mathcal{F}, P), B_{z}(r)$ a $\mathcal{T}_{c^{-}}$-closed ball in $E, y \in E \backslash B_{z}(r)$. Define the $L^{0}(\mathcal{F})$-convex hull of $\{y\} \cup B_{z}(r)$ by

$$
D(z, r, y):=\left\{t b+(1-t) y: b \in B_{z}(r), t \in L_{+}^{0}(\mathcal{F}) \text { and } 0 \leq t \leq 1\right\} .
$$


Definition 3.3 Let $(E,\|\cdot\|)$ be an $R N$ module over $R$ with base $(\Omega, \mathcal{F}, P), \leq_{1}$ and $\leq_{2}$ be both orderings on $E$. Then $\leq_{2}$ is finer than $\leq_{1}$, if

$$
x \leq_{2} y \Rightarrow x \leq_{1} y \text {. }
$$

In [3], we established the precise form of the Ekeland variational principle on a $\mathcal{T}_{c^{-}}$ complete $R N$-module. Here we only need its general form as follows.

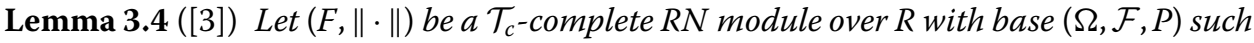
that $F$ has the countable concatenation property, $\varphi: F \rightarrow \bar{L}^{0}(\mathcal{F})$ have the local property. If $G \subset F$ is a $\mathcal{T}_{c}$-closed subset with the countable concatenation property and $\left.\varphi\right|_{G}$ is proper, $\mathcal{T}_{c}$-lower semicontinuous, and bounded from below on $G$, then for each point $x_{0} \in \operatorname{dom}\left(\left.\varphi\right|_{G}\right)$, there exists $z \in G$ such that the following are satisfied:

(1) $\varphi(z) \leq \varphi\left(x_{0}\right)-\left\|z-x_{0}\right\|$;

(2) for each $x \in G$ such that $x \neq z, \varphi(x) \not \leq \varphi(z)-\|x-z\|$, which means that $z$ is a maximal element in $\left(G, \leq_{\varphi}\right)$.

Remark 3.5 ([3]) The ordering $\leq_{\varphi}$ on $F$ is defined as follows: $x \leq_{\varphi} y$ if and only if either $x=y$, or $x$ and $y \in \operatorname{dom}(\varphi)$ are such that $\|x-y\| \leq \varphi(x)-\varphi(y)$.

Theorem 3.6 Let $(X,\|\cdot\|)$ be a $\mathcal{T}_{c}$-complete $R N$ module over $R$ with base $(\Omega, \mathcal{F}, P)$ such that $X$ has the countable concatenation property, $F \subset X$ a $\mathcal{T}_{c}$-closed subset with the countable concatenation property, and $z \in X \backslash F$. Let $r, R, \rho \in L_{++}^{0}(\mathcal{F})$ with $0<r<R<\rho$ on $\Omega$, then there exists $x_{0} \in \partial_{c}(F)$ such that

$$
\left\|x_{0}-z\right\| \leq \rho
$$

and

$$
D\left(z, r, x_{0}\right) \cap F=\left\{x_{0}\right\}
$$

where $R:=\bigwedge\{\|z-a\|: a \in F\}$, and $\partial_{c}(F)$ denotes the $\mathcal{T}_{c}$-boundary of $F$.

Proof We can, without loss of generality, suppose $z=0$.

Let $E:=F \cap B_{0}(\rho)$.

Define an ordering $\tilde{\leq}$ on $E$ as follows: $x_{1} \tilde{\leq} x_{2}$ if and only if $x_{2} \in D\left(0, r, x_{1}\right)$. It is easy to check that $\tilde{\leq}$ is a partial ordering.

Define a function $\varphi: E \rightarrow L_{+}^{0}(\mathcal{F})$ by $\varphi(x)=(\rho+r)(R-r)^{-1}\|x\|, \forall x \in E$.

Since $F$ and $B_{0}(\rho)$ are $\mathcal{T}_{c}$-closed and have the countable concatenation property, it follows that $E$ is $\mathcal{T}_{c}$-closed and has the countable concatenation property.

For each $A \in \mathcal{F}$, one can have

$$
\tilde{I}_{A} \cdot \varphi(x)=\tilde{I}_{A} \cdot(\rho+r)(R-r)^{-1}\|x\|=\tilde{I}_{A} \cdot(\rho+r)(R-r)^{-1}\left\|\tilde{I}_{A} \cdot x\right\|=\tilde{I}_{A} \cdot \varphi\left(\tilde{I}_{A} \cdot x\right), \quad \forall x \in E,
$$

which implies that $\varphi$ has the local property.

Since $\varphi$ is $\mathcal{T}_{c}$-continuous, it is easy to see that $\varphi$ is $\mathcal{T}_{c}$-lower semicontinuous.

Then from Lemma 3.4, there exists a maximal element $x_{0}$ in $\left(E, \leq_{\varphi}\right)$. 
We now prove that $\tilde{\leq}$ is finer than the ordering $\leq_{\varphi}$.

Let $x_{1}, x_{2}$ be points in $E$ such that $x_{1} \tilde{\leq} x_{2}$. Then one can have $x_{2} \in D\left(0, r, x_{1}\right)$; thus we can suppose

$$
x_{2}=(1-t) x_{1}+t v
$$

where $t \in L_{+}^{0}(\mathcal{F}), 0 \leq t \leq 1$, and $v \in B_{0}(r)$.

From (1), it follows that $\left\|x_{2}\right\| \leq(1-t)\left\|x_{1}\right\|+t\|v\|$, which implies

$$
t\left(\left\|x_{1}\right\|-\|v\|\right) \leq\left\|x_{1}\right\|-\left\|x_{2}\right\| .
$$

From $R-r \leq\left\|x_{1}\right\|-\|v\|$, one can have

$$
t \leq\left(\left\|x_{1}\right\|-\left\|x_{2}\right\|\right)(R-r)^{-1}
$$

Thus by (2) we have

$$
\begin{aligned}
\left\|x_{1}-x_{2}\right\| & =t\left\|v-x_{1}\right\| \leq t\left(\|v\|+\left\|x_{1}\right\|\right) \\
& \leq t(r+\rho) \leq(r+\rho)(R-r)^{-1}\left(\left\|x_{1}\right\|-\left\|x_{2}\right\|\right),
\end{aligned}
$$

which implies $x_{1} \leq_{\varphi} x_{2}$, and hence $\tilde{\leq}$ is finer than the ordering $\leq_{\varphi}$.

Since $x_{0}$ is a maximal element in $\left(E, \leq_{\varphi}\right)$ and $\tilde{\leq}$ is finer than $\leq_{\varphi}$, it is easy to check that $x_{0}$ is a maximal element in $(E, \tilde{\leq})$. Thus we have $\left\|x_{0}\right\| \leq \rho$ and $\left\{x_{0}\right\}=D\left(0, r, x_{0}\right) \cap E$, which implies $D\left(0, r, x_{0}\right) \cap F=\left\{x_{0}\right\}$.

For each $x \in D\left(0, r, x_{0}\right)$, we can suppose $x=t x_{0}+(1-t) v$, where $v \in B_{0}(r)$. Thus we have

$$
\|x\|=\left\|t x_{0}+(1-t) v\right\| \leq t\left\|x_{0}\right\|+(1-t)\|v\| \leq t \rho+(1-t) r<\rho
$$

on $\Omega$.

It is easy to see that for any $y \in F \backslash E$, $\|y\| \leq \rho$ does not hold. Hence we have $x_{0} \in \partial_{c}(F)$.

Remark 3.7 When the base space $(\Omega, \mathcal{F}, P)$ of the $R N$ module is trivial, namely $\mathcal{F}=\{\emptyset, \Omega\}$, our result automatically degenerates to the classical Daneš theorem. So our result is a nontrivial random extension.

Competing interests

The author declares to have no competing interests.

\section{Acknowledgements}

This work was supported by the 'New Start' Academic Research Projects of Beijing Union University (No. Zk10201304).

Received: 11 May 2014 Accepted: 23 July 2014 Published: 21 Aug 2014

\section{References}

1. Daneš, J: A geometric theorem useful in nonlinear functional analysis. Boll. Unione Mat. Ital. 6, 369-375 (1972)

2. Brøndsted, A: On a lemma of Bishop and Phelps. Pac. J. Math. 55, 335-341 (1974)

3. Guo, $T X$, Yang, YJ: Ekeland's variational principle for an $\bar{L}^{0}$-valued function on a complete random metric space. J. Math. Anal. Appl. 389, 1-14 (2012) 
4. Guo, TX, Xiao, HX, Chen, XX: A basic strict separation theorem in random locally convex modules. Nonlinear Anal. 71, 3794-3804 (2009)

5. Guo, TX, Li, SB: The James theorem in complete random normed modules. J. Math. Anal. Appl. 308, $257-265$ (2005)

6. Guo, TX, Shi, G: The algebraic structure of finitely generated $L^{0}(\mathcal{F}, K)$-modules and the Helly theorem in random normed modules. J. Math. Anal. Appl. 381, 833-842 (2011)

7. Zhao, SN, Guo, TX: The random reflexivities of complete random normed modules. Int. J. Math. (2011). doi:10.1142/S0129167X12500474

8. Tang, YH: A new version of the Gleason-Kahane-Zklazko theorem in complete random normed algebras. J. Inequal. Appl. (2012). doi:10.1186/1029-242X-2012-85

9. Guo, TX: Recent progress in random metric theory and its applications to conditional risk measures. Sci. China Ser. A 54, 633-660 (2011)

10. Guo, TX: Relations between some basic results derived from two kinds of topologies for a random locally convex module. J. Funct. Anal. 258, 3024-3047 (2010)

11. Tang, YH: The Wintner theorem in unital complete random normed algebras. Bull. Korean Math. Soc. 50(6), 1973-1979 (2013)

12. Tang, $\mathrm{YH}$ : Random spectral theorems of self-adjoint random linear operators on complete complex random inner product modules. Linear Multilinear Algebra 61(3), 409-416 (2013)

13. Tang, YH, Guo, TX: Complete random normed algebras. Southeast Asian Bull. Math. 37, 931-940 (2013)

14. Yang, YJ: Drop theorem and petal theorem in complete random normed modules. Acta Anal. Funct. Appl. 14(4), 411-418 (2012)

15. Guo, TX: Some basic theories of random normed linear spaces and random inner product spaces. Acta Anal. Funct. Appl. 1(2), 160-184 (1999)

16. Filipović, D, Kupper, M, Vogelpoth, N: Separation and duality in locally $L^{0}$-convex modules. J. Funct. Anal. 256, 3996-4029 (2009)

17. Göpfert, A, Tammer, C, Zălinescu, C: On the vectorial Ekeland's variational principle and minimal points in product spaces. Nonlinear Anal. 39, 909-922 (2000)

18. Finet, C, Quarta, L, Troestler, C: Vector-valued variational principles. Nonlinear Anal. 52, 197-218 (2003)

19. Araya, Y: Ekeland's variational principle and its equivalent theorems in vector optimization. J. Math. Anal. Appl. 346, 9-16 (2008)

10.1186/1029-242X-2014-317

Cite this article as: Yang: Daneš theorem in complete random normed modules. Journal of Inequalities and

Applications 2014, 2014:317

\section{Submit your manuscript to a SpringerOpen ${ }^{\circ}$ journal and benefit from:}

- Convenient online submission

- Rigorous peer review

- Immediate publication on acceptance

Open access: articles freely available online

- High visibility within the field

- Retaining the copyright to your article 